\title{
Optimum length of multimode optical branching waveguide for reducing its mode dependence
}

\author{
Y. Kokubun, T. Fuse, and K. Iga
}

\begin{abstract}
In this paper, we theoretically analyze the loss and branching ratio of multimode optical branching waveguides by using a ray tracing method to obtain less mode-dependent branching characteristics. First, a $2 \times 2$ optical mixing and branching waveguide with a step-index profile are considered. It is found that an optimum length to achieve a low loss and stable branching ratio exists. Second, a waveguide with mode scrambler is investigated. By sacrificing some amount of insertion loss, the mode dependence can be minimized.
\end{abstract}

\section{Introduction}

Multimode optical branching circuits and access devices are important in sophisticated optical communication network systems such as subscriber systems and local area networks. Several types of optical branching device such as microlens, ${ }^{1}$ fiber, ${ }^{2}$ and waveguide ${ }^{3-6}$ devices have been reported. In particular, the optical waveguide-type devices seem promising because of their inherent low loss and the potential for mass fabrication with good reproducibility. A main problem, however, is that the branching ratio at the $Y$-junction is not always unity because of its dependence on mode distribution.

To eliminate this problem, we have proposed a new waveguide mode scrambler integrated with mixing and branching waveguides. ${ }^{7}$ We fabricated this mode scrambler by using the electromigration technique ${ }^{8}$ and obtained a stable branching ratio. ${ }^{7}$ However, an optimum waveguide design to minimize the loss and device size has not been studied.

On the other hand, for conventional mixing and branching waveguides with a straight mixing region, the optimum design was not fully investigated either.

\footnotetext{
When this work was done Y. Kokubun and T. Fuse are with Yokohama National University, Faculty of Engineering, Division of Electrical \& Computer Engineering, 156 Tokiwadai, Hodogayaku, Yokohama 240, Japan; T. Fuse is now with Toshiba Corporation, VLSI Research Center, Saiwaiku, Kawasaki 210, Japan. K. Iga is with Tokyo Institute of Technology, Research Laboratory of Precision Machinery and Electronics, 4259 Nagatsuta, Midoriku, Yokohama 227, Japan.

Received 2 February 1985

0003-6935/85/234408-06\$02.00/0.

(C) 1985 Optical Society of America.
}

Thus there remains the possibility of obtaining stable branching characteristics with low loss by the conventional mixing and branching waveguide.

In this study, we investigate the branching characteristics of multimode waveguides to obtain optimum design conditions. We have used a ray tracing technique to analyze (1) tapered waveguides, (2) conventional mixing and branching waveguides with straight mixing region, and (3) waveguides with mode scramblers.

\section{Principle of Ray Tracing}

\section{A. Incident Condition}

Let us consider the case when a light source is coupled into a graded-index multimode optical fiber. After a sufficiently long distance propagation in the fiber, the mode distribution in the fiber would reach a steady state with a definite mode distribution, which can be approximated by a uniform mode distribution. The output end of the fiber is butt-jointed to the input end of a $2 \times 2$ multimode mixing and branching waveguide with a square cross section and step-index profile as shown in Fig. 1.

When we project the 3-D ray trajectory in a waveguide separately onto a vertical $Y-Z$ plane and a horizontal $X-Z$ plane, respectively, only the ray propagation in the $X-Z$ plane is concerned with the branching characteristics since the ray propagation in the $Y-Z$ plane repeats only the relection between the upper and lower claddings and has no influence on branching. Thus we consider only the projection of the 3-D ray trajectory on the 2-D $X-Z$ plane.

Figure 2 shows a light emittance profile at the end of a GI multimode fiber, which has a parabolic refractiveindex distribution given by Eq. (A1). The position and trend of a ray are normalized by the fiber core 


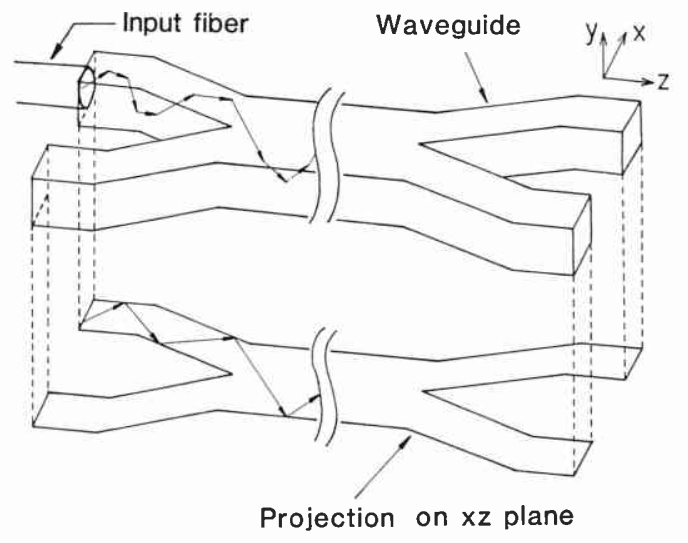

Fig. 1. Projection from 3-D ray trajectory to 2-D ray trajectory. GI fiber is butt-jointed to the branching waveguide with a square cross section.

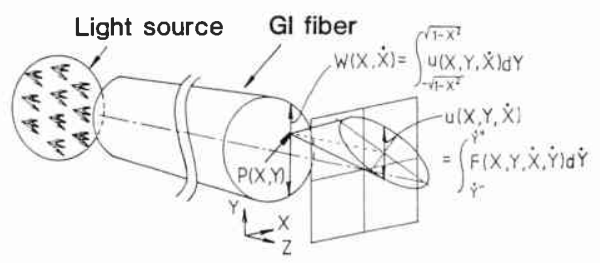

Fig. 2. Light emittance profile at the end of a GI multimode fiber.

radius $a$ and by the trend of ray with critical angle $\theta_{c}$, respectively, which are denoted by capital leters. For example, the $X$ components of position and trend with propagation angle $\theta$ are written by

$$
\left\{\begin{array}{l}
X=x / a \\
\dot{X}=\tan \theta / \tan \theta_{c}
\end{array}\right.
$$

Allowed values of $X$ and $\dot{X}$ emitted from the GI fiber must always satisfy the next condition (see the Appendix):

$$
X^{2}+\dot{X}^{2} \leq 1
$$

Since there exist many guided modes in a multimode fiber, we assume that the propagation modes are continuous, although actually they form a discrete set of eigenmodes. Here we image a cone extended from a point on the end of fiber and assume that the line which is connected with this point and the center of the fiber end corresponds to the $X$ axis. In terms of the direction along the $X$ axis, the extension angle is restricted from the condition of inequality (2), while in terms of the perpendicular direction to the $X$ axis, it does not suffer from any restriction. Therefore, the cross section of the cone will be elliptical. Thus the output rays are emitted from all points on the output end of a fiber, and the allowed region of the beam extends from a point $P(X, Y)$ constructing an elliptical cone as shown in Fig. 2. Since the rays which have the same $X$ and $\dot{X}$ and different $Y$ and $Y$ at the input end take the same trajectory in the $X-Z$ plane, we must take this degeneracy into account by introducing a weight function which can be derived from the integration of rays with respect to $Y$ and $\dot{Y}$. Thus we have the weight function using two solutions $\dot{Y}^{ \pm}$of Eq. (B1) as

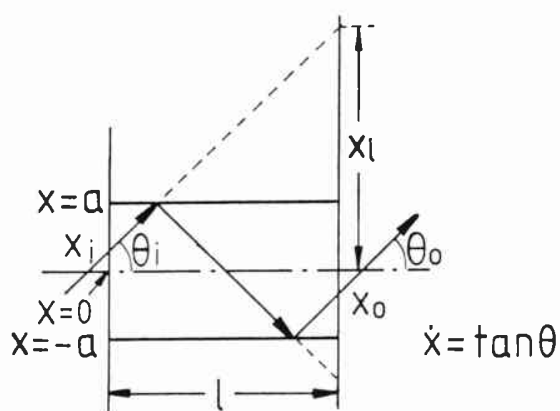

Fig. 3. Ray propagation in a straight waveguide (step-index type).

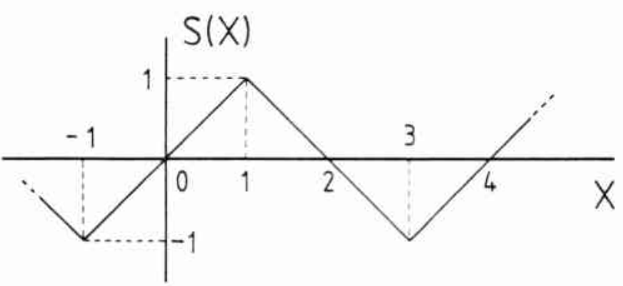

Fig. 4. Saw function $S(X)$.

$$
W(X, \dot{X})=\int_{-\sqrt{1-X^{2}}}^{\sqrt{1-X^{2}}} \int_{\dot{Y}^{-}}^{\dot{Y}^{+}} F(X, Y, \dot{X}, \dot{Y}) d \dot{Y} d Y,
$$

where $F(X, Y, \dot{X}, \dot{Y})$ is the density of rays.

If $F(X, Y, X, Y)$ is equal to 1 , which corresponds to uniform mode distribution like LED, we can easily integrate Eq. (3):

$$
W(X, \dot{X})=\pi\left(1-X^{2}-\dot{X}^{2}\right)^{1 / 2} .
$$

Using this weight function, we deal with a 3-D waveguide which has a square cross section.

\section{B. Ray Tracing of Step-Index Waveguides}

As for the projected branching waveguide in Fig. 1, we determine the position and trend of each ray on the input and output ends of the waveguide, which is divided into straight and bent parts.

Figure 3 shows the ray propagation in a step-index slab straight waveguide. Here we define the normalized position and trend of input and output rays as $X_{i}$, $\dot{X}_{i}, X_{O}$, and $\dot{X}_{O}$, respectively. Furthermore, $X_{l}$ is the hypothetical $X$ assuming that the ray propagates through a distance $l$ along the beam axis without reflection from the waveguide walls. The relation of $X_{i}$, $\dot{X}_{i}, X_{O}$, and $\dot{X}_{O}$ can be written as

$$
\left\{\begin{array}{l}
X_{o}=S\left(X_{l}\right), \\
\dot{X}_{o}=\dot{X}_{i}(-1)^{\left[\left|X_{l}+1\right| / 2\right],} \\
X_{l}=X_{i}+\dot{X}_{i}(l / a) \tan \theta_{c},
\end{array}\right.
$$

where $[X]$ is the Gaussian sign which means the integer part of $X$, and $S$ is the saw function of which the range is from -1 to 1 as shown in Fig. 4.

Figure 5 shows a propagating ray in the waveguide at a bent portion. Although the axis of the waveguide is bent, it can be assumed that only the propagation angle is changed with the position of the ray remaining 


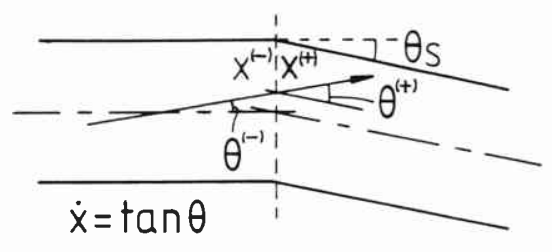

Fig. 5. Ray propagation in a bent waveguide.

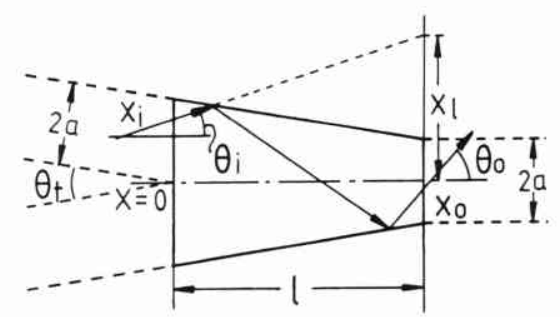

Fig. 6. Ray propagation in a tapered waveguide.

unaltered. Therefore, when we express the ray in the coordinate of bent waveguide, we obtain the ray equations at the bend:

$$
\left\{\begin{array}{l}
X^{(+)}=X^{(-1)} \\
\dot{X}^{(+)}=\dot{X}^{(-)}+\delta \dot{X} \\
\delta \dot{X}=\tan \theta_{s} / \tan \theta_{c}
\end{array}\right.
$$

where the superscripts (-) and (+) designate the ray before and after the bend, respectively, and we used the approximation that for $\theta_{s} \ll 1$

$$
\tan \left(\theta \pm \theta_{s}\right) \simeq \tan \theta \pm \tan \theta_{s}
$$

For a tapered waveguide, we use the same idea as mentioned above. The core width of the imput side is always twice that of the output side, and $X_{l}$ is defined similarly for a straight waveguide, as shown in Fig. 6. Since the propagation angle of a ray increases by tapered angle $\theta_{t}$ whenever it is reflected from a waveguide wall, we obtain

$$
\left\{\begin{aligned}
X_{o}= & S\left(X_{l}\right), \\
\dot{X}_{o}= & \left\{\dot{X}_{i}+\operatorname{sgn}\left(X_{i}\right) \cdot \tan \left(\theta_{t} \cdot\left[\left|X_{l}+1\right| / 2\right]\right) / \tan \theta_{c}\right\} \\
& \cdot(-1)^{\left[\left|X_{l}+1\right| / 2\right]}, \\
\dot{X}_{l}= & X_{i}+\dot{X}_{i}(l / a) \tan \theta_{c},
\end{aligned}\right.
$$

where $\operatorname{sgn}(X)$ expresses the sign of $X$.

\section{Estimation of Branching Characteristics}

Connecting the straight and bent parts, we trace each ray trajectory emitted from the GI fiber model. In our simulation, both the position and trend are divided equally into 101 parts. The rays whose propagation angle exceeds the critical angle $\theta c$ (i.e., $X>1$ ) are radiated out from the waveguide core, and these are regarded as radiation loss $\left(I_{r a d}\right)$. In the case of the branching waveguide, the branching ratio $R_{b}$ and radiation loss $\alpha$ are calculated in terms of input power $I_{t}$, two output powers $I_{l}$ and $I_{r}$, and radiation power $I_{r a d}$ as

$$
\begin{aligned}
R_{b} & =I_{l} / I_{r}, \\
\alpha & =I_{r a d} / I_{t}=-10 \log \left[\left(I_{r}+I_{l}\right) / I_{t}\right][\mathrm{dB}],
\end{aligned}
$$

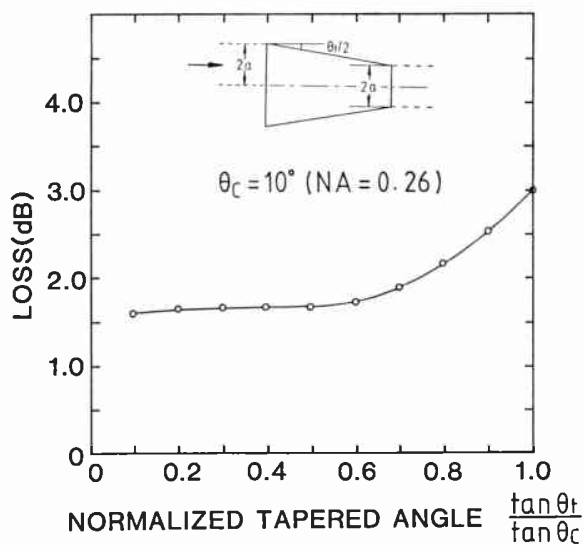

Fig. 7. Loss vs normalized tapered (branching) angle of tapered waveguide.

where we do not consider the coupling loss which is caused by the mismatch of the cross sections between the output fibers and the branching waveguides. That is, output power is counted as the quantity of rays with weight function given by Eq. (4), which can reach to the output ends of waveguide.

\section{Results}

\section{A. Radiation Loss of the Tapered Waveguide}

We simulated the radiation loss at the $Y$-junction with a tapered section. It is assumed that GI fiber is butt-jointed perpendicularly to the input surface of the 3-D tapered waveguide so that the edge of the fiber core is tangent to the inner edge of the waveguide core. The radiation loss is produced in the mixing taper since the propagation angle is forced to increase as the ray is reflected.

Figure 7 shows the loss vs tapered (branching) angle normalized by the critical angle of the waveguide. We selected the critical angle $\theta_{c}$ of $10^{\circ}$ for which the numerical aperture (N.A.) is to be 0.26 . The loss is $\sim 1.65$ $\mathrm{dB}$ and is almost constant in the range of $0.1-0.6$. (Most of the actual tapered angles seem to lie in this range.) This seems to be caused by the fact that the change in the propagation angle of rays by one reflection decreases with a decrease of the tapered angle, while the smaller tapered angle results in a longer propagation length. Thus the total change of propagation angle is almost constant, and the loss does not depend on the tapered angle. If the tapered angle approaches critical angle $\theta_{c}$, the number of rays which is to be radiated by an increase of a few reflections. Therefore, the loss increases linearly as the normalized tapered angle exceeds 0.6 , as shown in the figure. If we select the critical angle to be smaller than $10^{\circ}$, the range of the applicable tapered angle is expected to be narrow.

\section{B. Branching Characteristics of a $2 \times 2$ Mixing and Branching Waveguide}

To reduce the radiation loss in the $Y$-junction as mentioned above, the width of the mixing waveguide is assumed to be twice that of the branching waveguide. 
$\mathrm{NA}=0.26$

$\theta_{\mathrm{b}}=4.0$

$\mathrm{L} / 2 \mathrm{~A}=21.0$

Input $\bigsqcup_{-Z}^{X} X: Z=10: 3 \quad$ Output

Fig. 8. Ray trajectories in $2 \times 2$ mixing and branching waveguide with a straight waveguide (projected on the $X-Z$ plane).

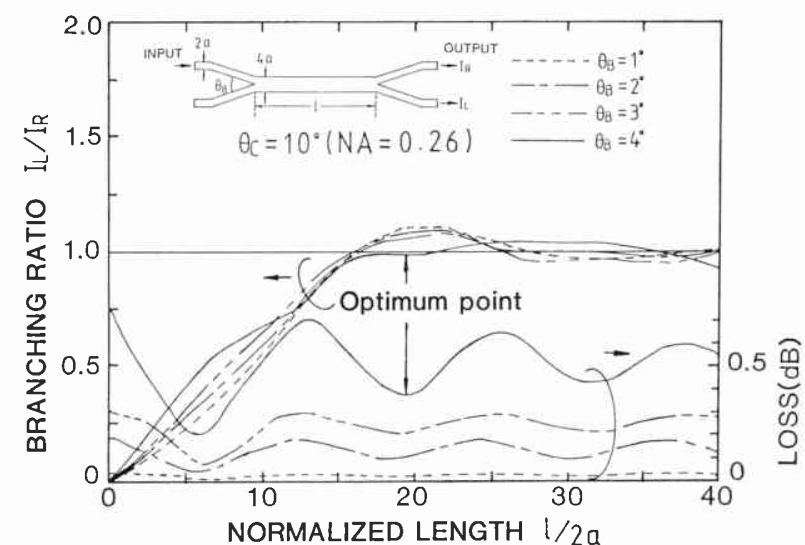

(a)

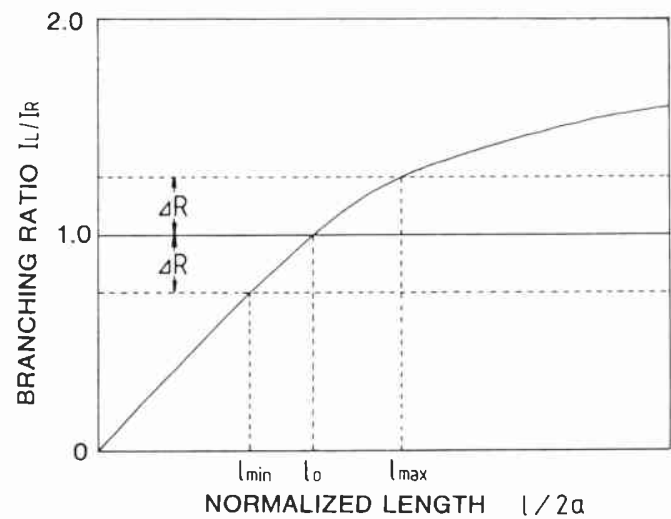

(b)

Fig. 9. Branching characteristics against normalized mixing length ( $2 \times 2$ mixing and branching waveguide with a straight waveguide):

(a) calculation; (b) $l_{\max }, l_{\min }, l_{\mathrm{o}}$ and $\Delta R$ are defined in this figure.

We traced some ray trajectories in a $2 \times 2$ mixing and branching waveguide with a conventional straight waveguide and investigated the branching characteristics of this type of waveguide.

Figure 8 shows these ray trajectories that are projected on the $X-Z$ plane. The branching angle is $4^{\circ}$, and the mixing length normalized by half of the mixing core width is 21 , a nominal value, and N.A. is assumed to be 0.26 . The scale of longitudinal direction is reduced to 0.3 of that of the transverse direction. It is seen that the rays emitted from the GI fiber, which have the normalized input position $X$ and trend $\dot{X}$

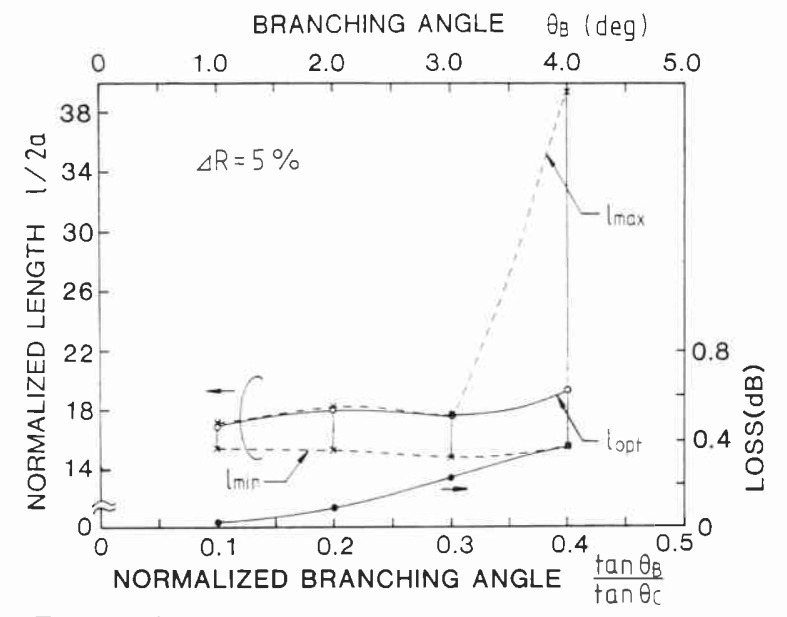

Fig. 10. $l_{\max }, l_{\min }$, and $l_{\text {opt }}$ vs normalized branching angle.

satisfying the condition given by Eq. (2), are distributed in two output ports.

Figure 9 shows the branching ratio and loss vs the normalized length of the mixing waveguide. It is seen that the branching ratio and radiation loss depend on waveguide parameters such as mixing length and branching angle. We define the fluctuation of the branching ratio as $\pm \Delta R$ as shown in Fig. $9(\mathrm{~b}) . \quad l_{\min }$ and $l_{\text {max }}$ are the minimum and maximum values of the normalized mixing length in the region $2 \Delta R$, respectively, and $l_{0}$ represents the first length we can obtain with a $1: 1$ branching ratio. Although the values of $l_{\mathrm{o}}$ is $\sim 16$, independent of the branching angle, the loss is not minimum at this value. We hence define the optimum value of normalized mixing length as $l_{\text {opt }}$ by which one can obtain a minimum loss in the region $2 \Delta R$. Figure 10 shows the variations of $l_{\min }, l_{\max }$, and $l_{\text {opt }}$ vs the normalized branching angle. At the branching angle of $4^{\circ}$, the tolerance of normalized mixing length (which is half of the difference between $l_{\text {max }}$ and $\left.l_{\text {min }}\right)$ is larger than that at an angle of $1-3^{\circ}$. The smaller the branching angle is, the longer the device size is. Thus we determine the optimum branching angle as $4^{\circ}$. To obtain a 1:1 stable branching ratio and minimum loss, the normalized mixing length is to be 19.4 from Fig. 9(a) when the branching angle is $4^{\circ}$.

It is desirable that the branching ratio not vary when we change the input conditions, such as position or 


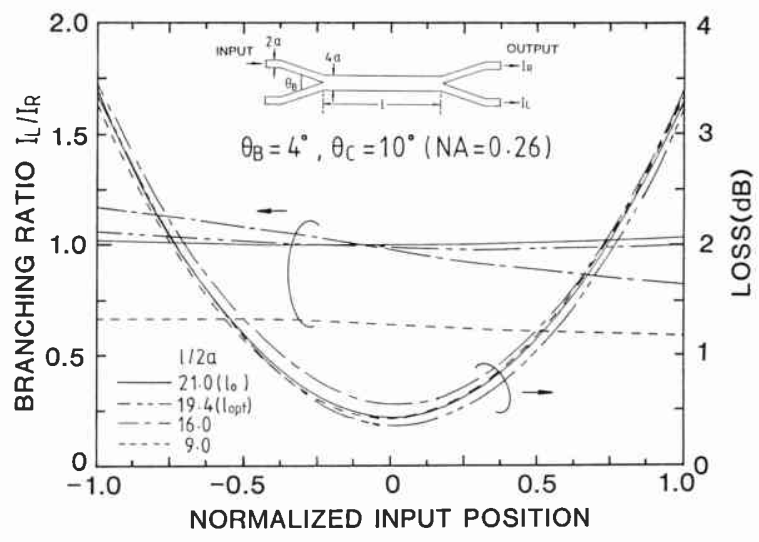

Fig. 11. Branching characteristics against input position $(2 \times 2$ mixing and branching waveguide with a straight waveguide).

angle of input fiber. Therefore, for several waveguide parameters including $l_{\text {opt }}$ and $l_{\text {or }}$, we simulated the branching characteristic vs normalized input position (Fig. 11). The branching ratio is stable over all input positions at the optimum value.

\section{Branching Characteristic of a Mode Scrambler}

To obtain optimum design conditions for our waveguide mode scrambler, we also investigated the dependence of the waveguide parameter on the branching characterisitcs by the same ray tracing technique. This mode scrambler consists of builtin zigzag waveguides connected alternately with a small bend angle as shown in Fig. 12. It reduces the branching ratio's dependence on mode distribution by converting modes at portions of the scrambling bend.

Figure 13 shows the branching ratio and loss vs bend number. We selected a branching angle of $4^{\circ}$, a bend angle of $2^{\circ}$, an N.A. of 0.26 , and a pitch of 12 , which is the normalized periodic length of a mode scrambler. The branching ratio has severe fluctuations dependent on bend number $M$ and approaches 1:1 although the loss increases slightly when $M$ becomes large. For a small bend number, the branching characteristic is asymmetric between the right input case and left input case because of the asymmetric structure of the mode scrambler as shown in Fig. 12. To obtain 1:1 stable branching ratio, the minimum number of scrambling bends should be 11 with $\Delta R$ equal to $\pm 5 \%$, resulting in $<1.0 \mathrm{~dB}$ of insertion loss.

Utilizing the asymmetric branching characteristics, furthermore, there is an ample possibility of realizing a new optical device in which the output power from a particular port is always higher compared with the other ports.

\section{Conclusion}

We have numerically analyzed the radiation loss at the $Y$-junction and branching characteristics of a $2 \times 2$ multimode mixing and branching waveguide by using a ray tracing method. The simulated waveguide has a 3-D structure with a square cross section, and its refractive-index profile is of a step-index type. The

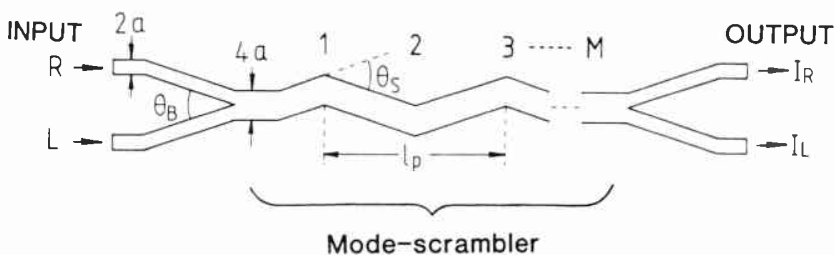

Fig. 12. Structure of the $2 \times 2$ branching waveguide with a mode scrambler.

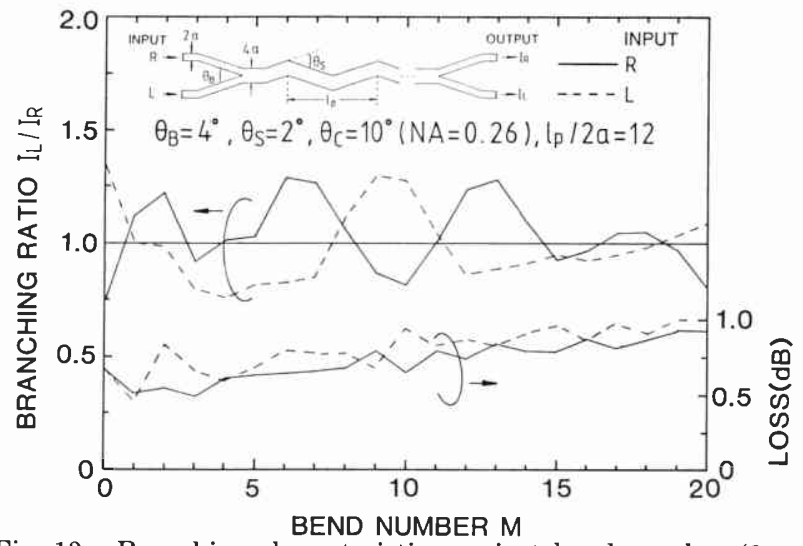

Fig. 13. Branching characteristics against bend number $(2 \times 2$ mixing and branching waveguide with a mode scrambler).

waveguide is assumed to be illuminated by a uniformly excited graded-index multimode fiber.

As a result, we have found that the branching ratio depends on the waveguide parameter, and there exists suitable length in a conventional straight mixing waveguide for the purpose of reducing the radiation loss at a $Y$-junction to which much attention has not been paid. We have also obtained a requirement of a waveguide mode scrambler for 1:1 branching, which results in less mode dependence by sacrificing an insertion loss of $\sim 1.0 \mathrm{~dB}$.

The ray tracing technique used here for a step-index waveguide could be extended to the analysis of gradedindex-type waveguides.

We would like to acknowledge Y. Suematsu, Tokyo Institute of Technology, and M. Toki and Y. Tsuzuki, Yokohama National University, for support. The authors also wish to thank T. Yamasaki, E. Okuda, T. Koizumi, and M. Oikawa, Nippon Sheet Glass Co. Ltd., for valuable suggestions and S. Misawa, S. Suzuki, and T. Baba for their help.

\section{Appendix A: Derivation of Inequality (2)}

We consider the ray trajectory in a cylindrical distributed index waveguide such as GI fiber, whose refractive-index distribution is given by

$$
n^{2}(r)=n^{2}(0)\left[1-(g r)^{2}\right],
$$

where $\left.r\left[=(x)^{2}+y^{2}\right)^{1 / 2}\right]$ is the radial distance from the center, and $g$ is a focusing constant. Treating the $x$ 


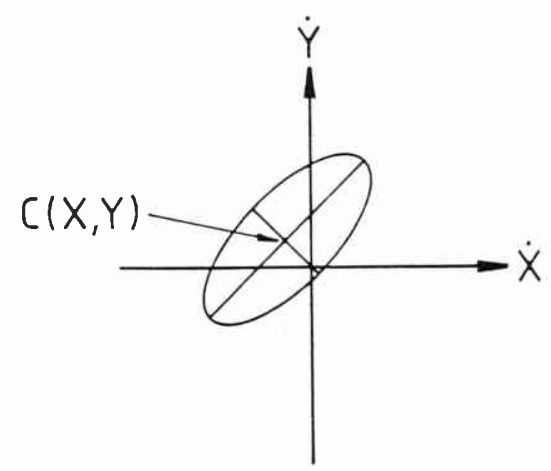

Fig. 14. Cross section of the elliptical cone

component for simplicity $r$ and using the ray equation, the position $x$ and angle $\theta$ of the ray at distance $z$ from the input end can be written as follows:

$$
\left\{\begin{array}{l}
x=x_{i} \cos (g z)+\left(\dot{x}_{i} / g\right) \sin (g z), \\
\dot{x}=-g x_{i} \sin (g z)+\dot{x}_{i} \cos (g z),
\end{array}\right.
$$

where $x_{i}$ is the position, and $\dot{x}_{i}=\tan \theta_{i}$ is the trend of the ray at the input end. We normalize $x$ and $x$ to the core radius and the trend of the critical angular ray as

$$
\left\{\begin{array}{l}
X=X_{i} \cos (g z)+X_{i} \sin (g z), \\
X=-X_{i} \sin (g z)+X_{i} \cos (g z),
\end{array}\right.
$$

where we used next approximation for paraxial rays,

$$
a g=\sin \theta_{\max } \simeq \tan \theta_{\max } .
$$

From Eq. (A3), we can obtain

$$
\left\{\begin{array}{l}
X=\sqrt{X_{i}^{2}+X_{i}^{2}} \cos (g z-\alpha), \\
X=-\sqrt{X_{i}^{2}+X_{i}^{2}} \sin (g z-\alpha), \\
\alpha=\cos ^{-1}\left(X_{i} / \sqrt{\left.X_{i}^{2}+X_{i}^{2}\right)} .\right.
\end{array}\right.
$$

Thus the allowed propagation ray in GI fiber is obtained from the condition

$$
X^{2}+\dot{X}^{2}=X_{i}^{2}+\dot{X}_{i}^{2} \leq 1 \text {. }
$$

\section{Appendix B: Derivation of the Elliptical Equation}

We show in Fig. 14 the cross section of the elliptical cone which is extended from the point $C(X, Y)$ and described on the $X-Y$ plane. This elliptical equation, which gives the relation of the position coordinates $X$ and $Y$ and the beam tends $\dot{X}$ and $\dot{Y}$, can be obtained geometrically as

$$
\left(I-Y^{2}\right) \dot{X}^{2}+\left(1-X^{2}\right) \dot{Y}^{2}+2 X Y \dot{X} \dot{Y}+X^{2}+Y^{2}-1=0 .
$$

Thus we obtain a quadratic equation with respect to $\dot{Y}$ :

$$
\left(1-X^{2}\right) \dot{Y}^{2}+2 X Y \dot{X} \dot{Y}+X^{2}\left(1-Y^{2}\right)+\dot{X}^{2}+Y^{2}-1=0 .
$$

\section{References}

1. M. Oikawa and K. Iga, "Stacked Array Components with Distributed-Index Planar Microlens," at Fourth Topical Meeting on Graded-Index Optical Imaging Systems (Kobe, 1983), paper H2.

2. E. G. Rawson and M. D. Bailey, "Bitaper Star Couplers with up to 100 Fiber Channels," Electron. Lett. 15, 432 (1979).

3. K. Nosu and R. Watanabe, "Slab Waveguide Star Coupler for Multimode Optical Fibers," Electron. Lett. 16, 608 (1980).

4. N. Takato and T. Kurokawa, "Polymer Waveguide Star Coupler," Appl. Opt. 21, 1940 (1982).

5. E. Okuda, I. Tanaka, and T. Yamasaki, "Planar Gradient-Index Glass Waveguide and its Applications to a 4-port Branched Circuit and Star Coupler," Appl. Opt. 23, 1745 (1984).

6. Y. Yamada, M. Kawachi, M. Yasu, and M. Kobayashi, "Fabrication of a High Silica Glass Waveguide Optical Accessor," Electron. Lett. 20, 589 (1984).

7. Y. Kokubun, S. Suzuki, T. Fuse, H. Uehara, K. Iga, M. Oikawa, and S. Misawa, "A Novel Mode Scrambler for Reducing Mode Dependence in Multimode Optical Waveguide," Electron. Lett. 19, 1009 (1983).

8. M. Oikawa, K. Iga, and T. Sanada, "Distributed-Index Planar Microlens Array Prepared from Deep Electromigration," Electron. Lett. 17, 452 (1981). 